Revista Brasileira de Agricultura Irrigada v.13, nº.3, p. 3454 - 3466, 2019

ISSN 1982-7679 (On-line)

Fortaleza, CE, INOVAGRI - http://www.inovagri.org.br

DOI: $10.7127 /$ rbai.v13n301066

Protocolo 1066.19 - 12/02/2019 Aprovado em 29/06/2019

\title{
CONDUTIVIDADE HIDRÁULICA EM FUNÇÃO DO CRESCIMENTO DE Urochloa decumbens
}

\author{
Anderson Takashi Hara ${ }^{1}$ João Vitor da Silva Domingues ${ }^{2}$, Antônio Carlos Andrade Gonçalves ${ }^{3}$, \\ Fernando André Silva Santos ${ }^{4}$, Heraldo Takao Hashiguti ${ }^{5}$, Roberto Rezende ${ }^{3}$
}

\section{RESUMO}

A cultura de Urochloa decumbens tem sido utilizada em sistemas de produção em plantio direto, com o objetivo de proporcionar melhorias em propriedades físico-hídricas do solo, ocasionando boas condições para o estabelecimento da cultura subsequente. Dentro das melhorias, destaca-se a possibilidade de modificar a variável condutividade hidráulica saturada do solo, possibilitando maiores taxas de infiltração, o que pode ser favorável ao desenvolvimento vegetal. Neste trabalho buscou-se avaliar, em um solo muito argiloso, o efeito promovido por um ciclo desta cultura sobre características físicas do solo, em uma escala compatível com as distâncias nas quais a interação planta e solo se estabelecem. Verificou-se que a condutividade hidráulica saturada média da camada de solo explorada pelo sistema radicular tornou-se três vezes maior ao final do ciclo da cultura. Identificou-se uma relação quadrática entre condutividade hidráulica e índice de área foliar da cultura, com um ponto de máximo na porção intermediária do ciclo, sugerindo que o volumoso sistema radicular, na porção final do ciclo, promove significativa redução da macroporosidade, o que se desfaz após a dessecação da cultura. Continuidade espacial foi identificada para as variáveis em estudo e constatou-se que a presença da cultura modifica o padrão espacial das mesmas, e que este padrão persiste no tempo, após a remoção da cultura.

Palavras-chave: geoestatística, semivariograma, semivariograma cruzado.

\section{HYDRAULIC CONDUCTIVITY IN THE FUNCTION OF GROWTH OF Urochloa decumbens}

\footnotetext{
ABSTRACT

The cultivation of Urochloa decumbens has been used in no-tillage system, with the objective of providing improvements in soil physical properties, causing good conditions for establishment of the subsequent crop. Among the improvements, the possibility of modifying the variable saturated hydraulic conductivity of the soil is highlighted, allowing higher rates of infiltration, which may be

${ }^{1}$ Doutor em Agronomia, Engenheiro Agrônomo, UEM, Maringá, PR. e-mail. haratakashi1987@gmail.com;

${ }^{2}$ Engenheiro Agrônomo, UEM, Maringá, PR. e-mail.jv_dominques@yahoo.com.br;

${ }^{3}$ Doutor, Professor, Engenheiro Agrícola, DAG, UEM, Maringá, PR. e-mail. goncalves.aca@gmail.com;

${ }^{4}$ Doutor, Professor, Engenheiro Agrônomo, UNEMAT, MT, e-mail. andresilva.com@gmail.com;

${ }^{5}$ Doutor, Professor, Engenheiro Agrônomo, FATECIE, Paranavaí, PR, e-mail. htakaoh@hotmail.com
} 
favorable to plant development. This work aimed to evaluate, in a clay soil, the effect promoted by a cycle of this crop on soil physical characteristics, in a scale compatible with the distances in which the interaction plant and soil are established. It was verified that the average saturated hydraulic conductivity of the soil layer explored by the root system became three times higher at the end of the crop cycle. It was identified a quadratic relation between hydraulic conductivity and leaf area index of the crop, with a maximum point in the intermediate portion of the cycle, suggesting that the massive root system, in the final portion of the cycle, promotes a significant reduction of macroporosity. which dissolves after desiccation of the culture. Spatial continuity was identified for the variables under study and it was verified that the presence of the culture modifies the spatial pattern of the same, and that this pattern persists in the time, after the removal of the culture.

Keywords: cross-semivariogram, geoestatistics, semivariogram.

\section{INTRODUÇÃO}

A cultura de Urochloa decumbens tem sido utilizada em sistemas de produção agrícola que envolvem preparo mínimo do solo, ou mesmo no sistema de plantio direto, com evidências de melhorias das condições físicohídricas do solo (COSTA et al., 2015; ZOLIN et al., 2016). As melhorias são justificadas pelo expressivo desenvolvimento do seu sistema radicular (GHESTEM et al., 2011; MORADI et al., 2011). O sistema radicular de uma cultura pode promover a formação de macroporos no solo por meio da morte de raízes (BEVEN; GERMANN, 1982), aumento da continuidade de poros (SCHOLL et al., 2014) e promover mudanças nos valores da condutividade hidráulica saturada do solo (BARTENS et al., 2008; ALLETTO et al., 2015).

A condutividade hidráulica saturada $(\mathrm{K})$ é uma variável que expressa a habilidade do meio em conduzir a água (MESQUITA; MORAES, 2004), relacionada com diversas variáveis físico-hídricas, como umidade no solo (VAN GENUCHTEN, 1980), porosidade do solo (GONÇALVES; LIBARDI, 2013), macroporosidade (MESQUITA; MORAES, 2004; SOUZA et al, 2014), densidade do solo (BISWAS; SI, 2009; JOSÉ et al., 2012).

Em vista dessas relações, essa variável tem sido o foco de estudos fitotécnicos buscando relações com o desenvolvimento vegetal (CALLEGARO, 2017), uma vez que a condutividade hidráulica, de certa forma, integra o efeito de variáveis físico-hídricas. Diante da relação solo-planta-água surge a hipótese de que o sistema radicular da cultura de Urochloa pode alterar as características do solo, modificando a sua capacidade de permitir movimentação de água no perfil. As alterações promovidas sobre a condutividade hidráulica saturada do solo interferem na magnitude dos seus valores e também no seu padrão de distribuição espacial, o que é persistente no tempo, após a remoção da cultura.

Diante do contexto, objetivou-se com este trabalho estabelecer a relação entre o desenvolvimento da cultura de Urochloa com variáveis hidráulicas do solo, umidade e condutividade hidráulica saturada, em um Nitossolo Vermelho durante um período de 500 dias.

\section{MATERIAL E MÉTODOS}

O experimento foi conduzido em uma área experimental cujo solo é classificado como Nitossolo Vermelho, de textura argilosa, da região noroeste do Estado do Paraná, conforme descrito em Hara et al. (2019).

Em fevereiro de 2016 o trabalho foi iniciado em uma condição na qual a cultura de Urochloa decumbens estava cobrindo uniformemente toda a área experimental, estando ali presente a mais de dois anos. Sondas de TDR com comprimento de $0,20 \mathrm{~m}$ foram instaladas verticalmente em 88 pontos experimentais demarcados na área de acordo com uma malha regular de amostragem, conforme Figura 1. 


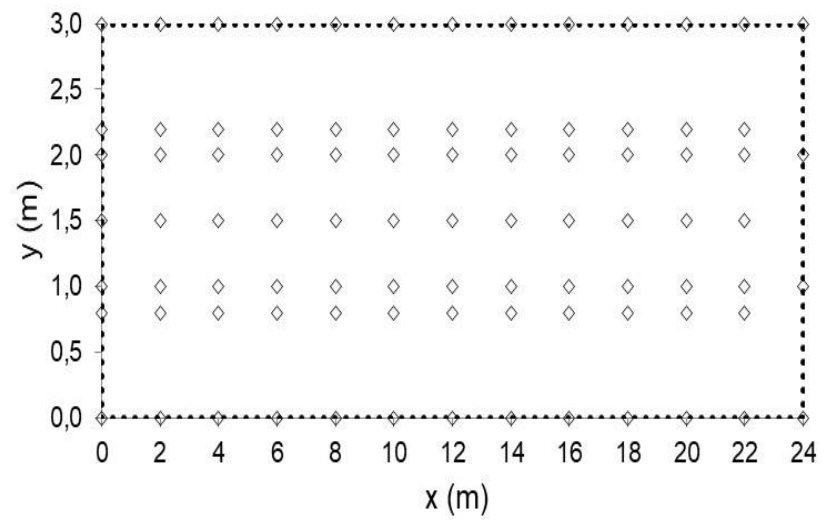

$\diamond$ Sistema de amostragem regular

Figura 1. Pontos de amostragem na área experimental.

Foi utilizado para a leitura das sondas de TDR, um osciloscópio da marca TRASE, modelo 6050X1 e, por meio de um modelo de calibração ajustado para este solo (TRINTINALHA, 2005) foi estimada a umidade do solo em base volume $(\Theta)$ na camada $0-0,20 \mathrm{~m}$.

No dia 5 de fevereiro de 2016 foi realizada a dessecação da cultura e foram instalados tubos de PVC de diâmetro externo de $0,05 \mathrm{~m}$, com comprimento de $0,15 \mathrm{~m}$, até uma profundidade de $0,03 \mathrm{~m}$ no solo, em cada ponto, distanciando-se cerca de $0,15 \mathrm{~m}$ da sonda de TDR, para que fossem feitas as medidas de condutividade hidráulica saturada do solo $(\mathrm{K})$, de acordo com a metodologia de Bagarello et al. (2004), na qual a avaliação de K é realizada adicionando-se uma lâmina de água de $0,05 \mathrm{~m}$ no interior do tubo e medindo-se o tempo necessário para a infiltração desta no solo. Uma hora antes das medidas de $\mathrm{K}$ foi estimada a umidade do solo nas 88 posições do sistema de amostragem regular, utilizando-se leituras de TDR. Os valores de tempo e umidade média do solo foram utilizados na Equação 1, para a determinação do correspondente valor de $\mathrm{K}$, em cada ponto.

O coeficiente $\alpha$ (ALPHA) da equação de van Genuchten foi obtido a partir do trabalho de Blainsk et al. (2009) e o valor médio de umidade do solo na saturação $\left(\theta_{s}\right)$ foi de 0,57 $\mathrm{m}^{3} \mathrm{~m}^{-3}$, para os 88 pontos de amostragem, nos quais foram coletadas amostras indeformadas de solo, em anel metálico de volume igual a 0,0001 $\mathrm{m}^{3}$ (HARA; GONÇALVES, 2018), no dia 23 de fevereiro de 2017.

$$
K=\frac{\Delta \theta}{(1-\Delta \theta) t_{a}}\left[\frac{D}{\Delta \theta}-\frac{\left(D+\frac{1}{\alpha}\right)}{(1-\Delta \theta} \ln \left(1+\frac{(1-\Delta \theta) D}{\Delta \theta\left(D+\frac{1}{\alpha}\right)}\right)\right]
$$

Em que: $K$ - Condutividade hidráulica saturada $\left(\mathrm{mm} \mathrm{s}^{-1}\right) ; \quad \theta \Delta$ - diferença de umidade do solo na condição de saturação e da umidade do solo anterior ao procedimento experimental; $t_{a}$ - tempo para a completa infiltração da lâmina adicionada (s); D - lâmina de água adicionada no infiltrômetro $(\mathrm{mm}) ; \alpha$ - coeficiente alfa da equação de Van Genuchten.

No dia 28 de março de 2016 foram observadas as primeiras plantas se estabelecendo na área. O estabelecimento da cultura ocorreu de forma espontânea, a partir das sementes presentes na área, oriundas dos 
ciclos de desenvolvimento anteriores da cultura. Estas datas foram estabelecidas tendo como critério aguardar o pleno desenvolvimento da cultura e, a partir daí, defasar as leituras em intervalos de aproximadamente um mês, até ao final, quando foram feitas duas leituras próximas entre si. As medidas de umidade do solo, assim como as de $\mathrm{K}$, foram efetuadas sem a cultura $(\mathrm{K} 1)$, em 04/03/16 e ao longo do seu crescimento subsequente (K2, K3, K4, K5, K6, K7 e K8), respectivamente nas datas 22/08/16, 02/10/16, 09/01/2017, 24/02/17, 29/03/2017, 28/04/17, 01/05/17. A série de valores K3 foi descartada por apresentar falhas metodológicas.

Na primeira semana de maio de 2017 foi realizada a dessecação da cultura com produto comercial com princípio ativo Glifosato, em dose de 2,0 $\mathrm{L} \mathrm{ha}^{-1}$, uma semana após a dessecação, leituras de umidade do solo e de K foram realizadas, nos dias 15/05/17, 26/05/17, 26/06/17, 30/06/17, 03/07/17, 10/07/17 e 17/07/17. Esses momentos foram referenciados, como K9, K10, K11, K12, K13, K14 e K15, respectivamente. A série de valores K11 foi descartada por apresentar falhas metodológicas. No total, decorreram 500 dias de experimento, nos dois ciclos de cultura avaliados. Os valores de umidade média no perfil do solo, na camada avaliada, entre 0 e $0,20 \mathrm{~m}$ de profundidade, em cada um dos 15 momentos definidos para as leituras, foram referenciados como $\Theta 1, \Theta 2, \Theta 3, \Theta 4, \Theta 5, \Theta 6$, Ө7, $98, \Theta 9, \Theta 10, \Theta 11, \Theta 12, \Theta 13, \Theta 14$ e $\Theta 15$, respectivamente. Durante o ciclo de desenvolvimento da cultura foram medidos os valores do índice de área foliar (IAF) em quatro (4) períodos, nas datas de 23/03/16, 15/12/16, $22 / 02 / 17$ e $22 / 04 / 17$. Para obter o IAF foram realizadas as coletas das folhas que foram delimitadas por um amostrador cilíndrico metálico de $0,30 \mathrm{~m}$ de altura e $0,113 \mathrm{~m}$ de diâmetro, nas 88 posições de amostragem (HARA et al., 2019). Posteriormente as folhas foram armazenadas em sacolas plásticas, para serem processadas no laboratório para a determinação da área foliar de acordo com a metodologia da imagem digital (HARA et al., 2019) utilizando o software QUANT. O IAF foi obtido pela razão da área foliar pela área interna do amostrador que foi utilizado para delimitar a coleta das folhas.

As análises estatísticas das variáveis abordadas foram realizadas, buscando-se avaliar como estas foram modificadas ao longo do ciclo da cultura e como ocorre a interação entre elas. Buscando uma melhor compreensão destas relações, foi feita uma abordagem geoestatística da distribuição dos valores destas variáveis no espaço, bem como de suas interrelações. Para fins de comparação entre semivariogramas, estes foram escalonados conforme metodologia apresentada por Hara e Gonçalves (2018). As estimativas de valores de semivariância e semivariância cruzada foram obtidas com o emprego do software VARIOWIN.

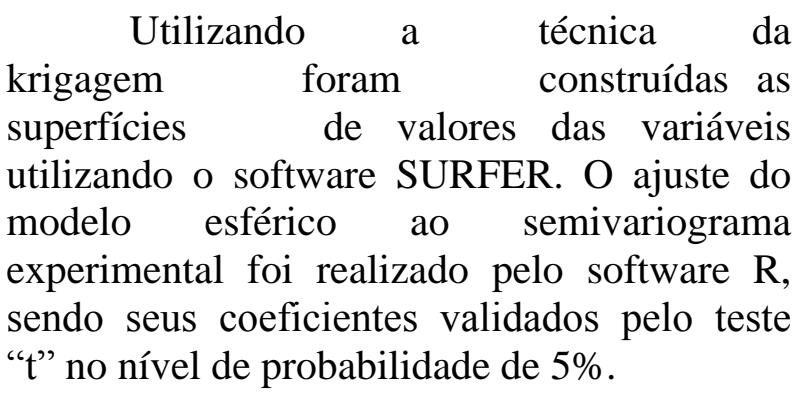

\section{RESULTADOS E DISCUSSÃO}

$\mathrm{Na}$ Tabela 1 são apresentados os momentos estatísticos para as 13 séries de dados de condutividade hidráulica saturada do solo (K), avaliadas durante a condução do experimento. Verifica-se o crescimento dos valores médios de $\mathrm{K}$ à medida que as plantas se desenvolvem, tornando-se cinco vezes maiores que na condição de solo sem cobertura vegetal. Isso evidencia $o$ forte impacto causado pelo desenvolvimento do sistema radicular das plantas sobre a capacidade do solo de permitir a infiltração de água. 
Tabela 1. Estatística descritiva, média (Méd), mediana (Med), mínimo (Mín), máximo (Máx), desvio padrão (DP), coeficiente de variação (CV), assimetria (Ass) e curtose (Cur) da condutividade hidráulica saturada $(\mathrm{K})$, nos diferentes momentos avaliados (Mom) em um Nitossolo Vermelho, para as condições de cobertura (Cob) viva e morta avaliados na área experimental

\begin{tabular}{|c|c|c|c|c|c|c|c|c|c|c|}
\hline \multirow{2}{*}{ Mom } & \multirow{2}{*}{ Data } & \multirow{2}{*}{ Cob } & Méd & Med & Mín & Máx & DP & \multirow{2}{*}{$\begin{array}{l}\mathrm{CV} \\
(\%)\end{array}$} & \multirow{2}{*}{ Ass } & \multirow{2}{*}{ Cur } \\
\hline & & & \multicolumn{5}{|c|}{----------------- $\mathrm{mm} \mathrm{h}^{-1}$---------------- } & & & \\
\hline K1 & $04 / 03 / 16$ & Viva & 22,81 & 4,82 & 0,0002 & 172,13 & 39,24 & 172,00 & 2,25 & 4,71 \\
\hline K2 & $22 / 08 / 16$ & Viva & 98,05 & 55,89 & 0,0690 & 782,43 & 139,04 & 141,80 & 3,31 & 13,24 \\
\hline K4 & $09 / 01 / 16$ & Viva & 114,01 & 67,46 & 0,2332 & 841,29 & 145,40 & 127,53 & 2,57 & 8,33 \\
\hline K5 & $24 / 01 / 17$ & Viva & 101,43 & 57,89 & 0,0429 & 563,92 & 120,60 & 118,91 & 1,94 & 4,06 \\
\hline K6 & $29 / 03 / 17$ & Viva & 72,25 & 45,62 & 0,0148 & 373,56 & 73,86 & 102,23 & 1,68 & 3,12 \\
\hline K7 & $28 / 04 / 17$ & Viva & 60,24 & 36,85 & 0,0078 & 268,66 & 64,80 & 107,57 & 1,35 & 1,21 \\
\hline K8 & $01 / 05 / 17$ & Viva & 61,66 & 27,75 & 0,0085 & 360,70 & 75,03 & 121,67 & 2,11 & 4,73 \\
\hline K9 & $15 / 05 / 17$ & Morta & 125,76 & 59,44 & 0,0014 & 808,41 & 161,45 & 128,37 & 2,09 & 4,70 \\
\hline K10 & $26 / 05 / 17$ & Morta & 80,89 & 54,14 & 0,0520 & 367,07 & 84,66 & 104,66 & 1,45 & 1,67 \\
\hline K12 & $26 / 06 / 17$ & Morta & 68,00 & 31,60 & 0,0043 & 340,08 & 84,59 & 124,40 & 1,70 & 2,03 \\
\hline K13 & $03 / 07 / 17$ & Morta & 52,58 & 25,80 & 0,0027 & 235,13 & 60,50 & 115,06 & 1,47 & 1,06 \\
\hline K14 & $10 / 07 / 17$ & Morta & 72,67 & 35,78 & 0,0004 & 397,84 & 89,05 & 122,54 & 1,80 & 2,88 \\
\hline K15 & $17 / 07 / 17$ & Morta & 78,70 & 41,59 & 0,0004 & 539,52 & 105,39 & 133,91 & 2,32 & 6,12 \\
\hline
\end{tabular}

A partir da série K5 pode-se observar a redução dos valores médios de $\mathrm{K}$ até $\mathrm{o}$ momento referente à série K8, após o que o herbicida foi aplicado promovendo a dessecação da cultura. Provavelmente esta redução está associada ao amplo desenvolvimento do sistema radicular cujo volume acarreta a ocupação de um percentual expressivo do espaço poroso do solo, em especial de macroporos que constituem as vias de infiltração da água no solo.

Decorridos cerca de 10 dias após a dessecação, foram obtidos os valores referentes à série K9. Pode-se verificar que o valor médio foi o mais elevado dentre todos os momentos avaliados. Isto sugere que a perda de água e consequente redução do volume do sistema radicular liberou grande parte do espaço poroso do solo para a infiltração de água, nestas condições. A partir desta condição, observa-se que os valores médios são reduzidos, estabilizando-se na ordem de $73 \mathrm{~mm} \mathrm{~h}^{-1}$, sinalizando que parte da macroporosidade do solo se rearranja, tendendo a uma estabilização. O efeito benéfico da atuação do sistema radicular da cultura, no entanto, permanece, uma vez que essa maior condutividade é da ordem de três vezes maior que o verificado antes do início do estabelecimento da cultura $\left(\mathrm{K} 1=23 \mathrm{~mm} \mathrm{~h}^{-1}\right)$.

Observa-se na Tabela 1, entretanto, que as distribuições de valores em cada série apresentam forte assimetria com alongamento da distribuição à direita, em função da presença de alguns valores muito elevados. Isto é evidenciado pelos coeficientes de assimetria e pelos valores de coeficiente de variação $(\mathrm{CV})$ sempre acima de $100 \%$, valores semelhantes ao encontrado por Scherpinsk et al. (2010). Também se pode verificar o afastamento entre os valores de média e mediana para as séries de valores.

Assim, foi feita uma transformação logarítmica dos dados, buscando uma maior simetria das distribuições, para as avaliações estatísticas feitas em seguida. Para tanto, cada valor foi multiplicado por 1000 e transformado com a função logarítmica natural, de forma a que os valores gerados fossem superiores à unidade. Na Tabela 2 estes valores são apresentados.

São apresentados na Tabela 2 os momentos estatísticos para as quatro séries de valores de IAF obtidos durante o ciclo da cultura, entre os momentos referentes às séries K1 a K8. Pode-se observar baixos valores dos 
coeficientes de assimetria, evidenciando as distribuições simétricas, o que é evidenciado pela proximidade entre os valores de média e mediana.

Tabela 2. Estatística descritiva, média (Méd), mediana (Med), mínimo (Mín), máximo (Máx), desvio padrão (DP), coeficiente de variação (CV), assimetria (Ass) e curtose (Cur) nos diferentes momentos avaliados (Mom) de valores de condutividade hidráulica saturada transformada $(\operatorname{Ln}(1000 \mathrm{~K}))$ avaliados no Nitossolo Vermelho, para as condições de cobertura (Cob) viva e morta e para os valores de índice de área foliar (IAF) em quatro momentos durante o ciclo da cultura avaliados na área experimental.

\begin{tabular}{|c|c|c|c|c|c|c|c|c|c|c|}
\hline \multirow{2}{*}{ Mom } & \multirow{2}{*}{ Data } & \multirow{2}{*}{ Cob } & Méd & Med & Mín & Máx & DP & \multirow{2}{*}{$\begin{array}{l}\mathrm{CV} \\
(\%)\end{array}$} & \multirow{2}{*}{ Ass } & \multirow{2}{*}{ Cur } \\
\hline & & & \multicolumn{5}{|c|}{----------- Ln $(1000 \mathrm{~K})$------------ } & & & \\
\hline K1 & $04 / 03 / 2016$ & Viva & 7,36 & 8,86 & 0,4 & 12,06 & 3,83 & 52,03 & 0,869 & 0,869 \\
\hline $\mathrm{K} 2$ & $22 / 08 / 2016$ & Viva & 10,59 & 10,93 & 4,23 & 13,57 & 1,68 & 15,84 & 0,364 & 0,364 \\
\hline $\mathrm{K} 4$ & 09/01/2016 & Viva & 10,83 & 11,12 & 5,45 & 13,64 & 1,52 & 14,04 & 0,332 & 0,332 \\
\hline K5 & $24 / 01 / 2017$ & Viva & 10,54 & 10,97 & 3,76 & 13,24 & 1,98 & 18,77 & 0,427 & 0,427 \\
\hline K6 & $29 / 03 / 2017$ & Viva & 10,52 & 10,73 & 2,69 & 12,83 & 1,51 & 14,36 & 0,332 & 0,332 \\
\hline K7 & $28 / 04 / 2017$ & Viva & 10,03 & 10,51 & 2,06 & 12,5 & 1,94 & 19,34 & 0,424 & 0,424 \\
\hline K8 & $01 / 05 / 2017$ & Viva & 10,22 & 10,23 & 2,14 & 12,8 & 1,68 & 16,4 & 0,357 & 0,357 \\
\hline K9 & $15 / 05 / 2017$ & Morta & 10,57 & 10,99 & 0,36 & 13,6 & 2,26 & 21,41 & 0,485 & 0,485 \\
\hline K10 & $26 / 05 / 2017$ & Morta & 10,46 & 10,9 & 3,95 & 12,81 & 1,78 & 17,05 & 0,385 & 0,385 \\
\hline $\mathrm{K} 12$ & $26 / 06 / 2017$ & Morta & 9,99 & 10,36 & 1,47 & 12,74 & 2,29 & 22,89 & 0,49 & 0,49 \\
\hline K13 & 03/07/2017 & Morta & 9,91 & 10,16 & 1,01 & 12,37 & 2,04 & 20,6 & 0,44 & 0,44 \\
\hline K14 & $10 / 07 / 2017$ & Morta & 10,36 & 10,56 & 2,86 & 12,89 & 1,71 & 16,47 & 0,373 & 0,373 \\
\hline \multirow[t]{2}{*}{ K15 } & 17/07/2017 & Morta & 10,18 & 10,66 & 2,81 & 13,2 & 2,09 & 20,57 & 0,457 & 0,457 \\
\hline & \multicolumn{10}{|c|}{---------------- $\mathrm{m}^{2} \mathrm{~m}^{-2}$--------------- } \\
\hline IAF1 & $23 / 03 / 2016$ & Viva & 0,579 & 0,62 & 0,077 & 0,949 & 0,222 & 38,43 & 0,047 & 0,047 \\
\hline IAF2 & $15 / 12 / 2016$ & Viva & 1,063 & 0,777 & 0,235 & 4,077 & 0,686 & 64,58 & 0,145 & 0,145 \\
\hline IAF3 & $22 / 02 / 2017$ & Viva & 1,545 & 1,449 & 0,193 & 3,518 & 0,701 & 45,34 & 0,149 & 0,149 \\
\hline IAF4 & $22 / 04 / 2017$ & Viva & 2,636 & 2,324 & 0,517 & 8,468 & 1,474 & 55,93 & 0,312 & 0,312 \\
\hline
\end{tabular}

As distribuições de IAF apresentam valores elevados de $\mathrm{CV}$ mas apresentam valores baixos para coeficiente de assimetria, possibilitando que fossem consideradas como simétricas para as análises posteriores.

Os valores médios de IAF crescem até um máximo de $2,64 \mathrm{~m}^{2} \mathrm{~m}^{-2}$, evidenciando o desenvolvimento da parte aérea das plantas durante este período do experimento. $\mathrm{Na}$ Figura 2 são mostrados os valores médios de IAF para as quatro avaliações realizadas e as barras correspondentes aos intervalos de confiança, no nível de $95 \%$ de probabilidade. Os valores são estatisticamente diferentes entre si, o que demonstra o crescimento da parte aérea da cultura, durante o período avaliado. 


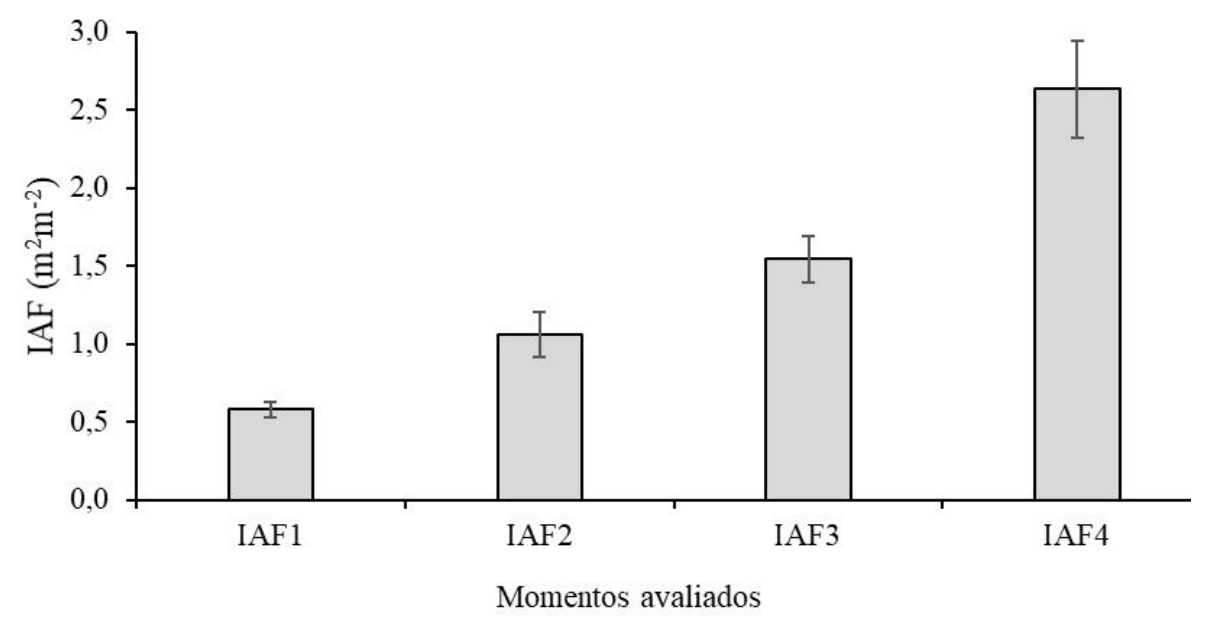

Figura 2. Valores médios de IAF medidos durante o ciclo da cultura e respectivos intervalos de confiança para $95 \%$ de probabilidade.

Na Figura 3A são mostrados os valores médios de Ln (1000K) para as séries estudadas e as barras referentes ao intervalo de confiança associado a cada média.
Pode-se verificar que os valores médios descrevem a expressiva elevação de valores de $\mathrm{K}$ com o desenvolvimento inicial da cultura.
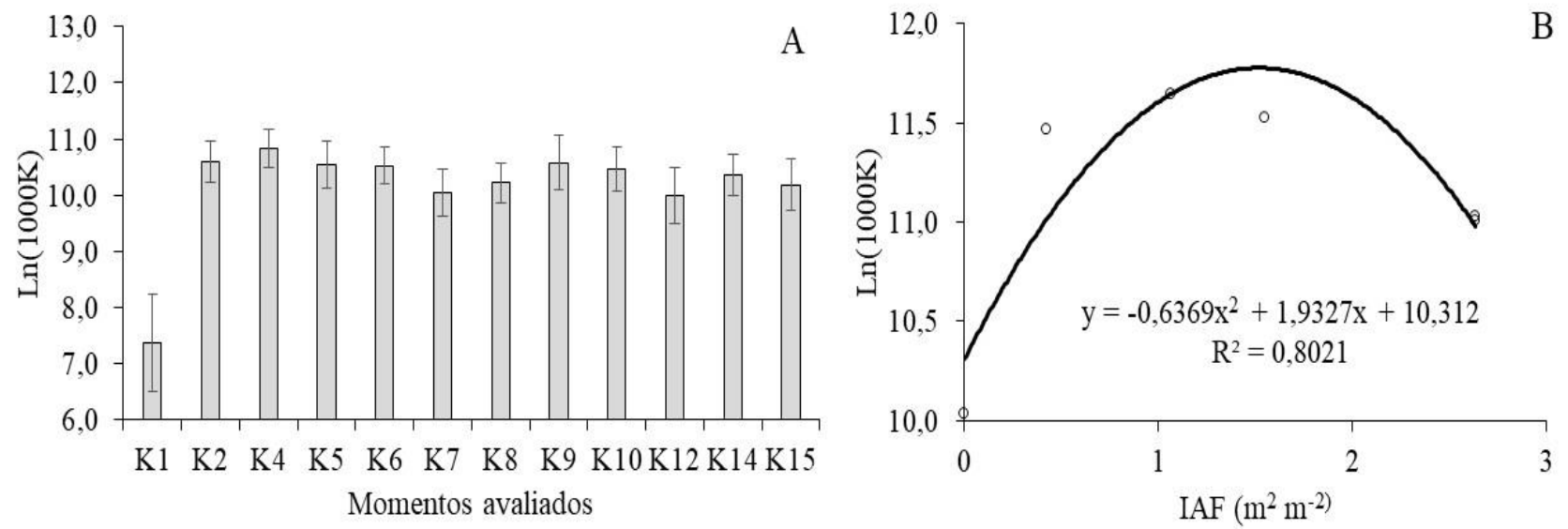

Figura 3. Valores médios dos dados transformados por $\operatorname{Ln}(1000 \mathrm{~K})$ e barras correspondentes ao intervalo de confiança em torno da média para $95 \%$ de probabilidade (A) e valores de $\operatorname{Ln}(1000 \mathrm{~K})$ e correspondentes valores de índice de área foliar (IAF) durante o ciclo (B).

No entanto, a variabilidade ainda elevada dos valores em cada série conduz a um intervalo de confiança muito amplo em todas elas, fazendo com que a diferenciação dos valores a partir de K2 fique comprometida. De acordo com (MOREIRA et al., 2012), a superposição dos intervalos de confiança acarreta a não diferença estatística dos valores médios, para o nível de probabilidade empregado, que foi de $5 \%$.

Ao longo do ciclo da cultura (K1 a K8) os valores de $\mathrm{K}$ variam de forma expressiva.
Na. Figura 3B são mostrados os valores médios de Ln $(1000 \mathrm{~K})$ em função dos valores de IAF medidos. A relação entre os valores destas variáveis pode ser descrita por um modelo quadrático com coeficiente de determinação de $84 \%$. Este modelo quadrático apresentou um ponto de máximo para IAF da ordem de $1,5 \mathrm{~m}^{2}$ $\mathrm{m}^{-2}$.

A partir do momento no qual a cultura começa a se desenvolver, o sistema radicular contribui para o crescimento da condutividade hidráulica saturada do solo, sendo este efeito 
crescente com o desenvolvimento da cultura, até o IAF de $1,5 \mathrm{~m}^{2} \mathrm{~m}^{-2}$.

A partir do momento em que o IAF correspondeu ao valor máximo de condutividade hidráulica saturada do solo, o desenvolvimento do sistema radicular começa a restringir o movimento da água, por ocupar uma porção cada vez mais expressiva da porosidade, reduzindo a capacidade do solo de permitir a infiltração de água. Nesta condição, porém, os valores de $\mathrm{K}$ permanecem muito superiores ao do solo antes da cobertura vegetal.

Pode-se verificar na Tabela 1 que a variabilidade de $\mathrm{K}$ com solo nu é mais elevada que com solo vegetado, o que é expresso pelo CV para a série K1 de $172 \%$, valor que é reduzido para próximo de $100 \%$, quando a cultura apresenta pleno desenvolvimento. Após a dessecação, o valor de CV tendeu a crescer novamente, atingido valores da ordem de $125 \%$, em média, mostrando que a ausência da cultura conduziu a maior variabilidade no espaço da variável $\mathrm{K}$.

$\mathrm{Na}$ Tabela 3 são apresentados os momentos estatísticos para as séries de valores de umidade do solo com base em volume, medidos no momento em que foram feitas as respectivas medidas de condutividade hidráulica saturada do solo. A observação dos valores mínimo e máximo revela que houve uma grande amplitude de valores de umidade em cada série, evidenciando a variabilidade natural do solo na área experimental. Em geral, as distribuições são razoavelmente simétricas, com coeficiente de variação entre 10 a $20 \%$, estando os maiores valores relacionados com a condição de solo mais seco.

Tabela 3. Estatística descritiva, média (Méd), mediana (Med), mínimo (Mín), máximo (Máx), desvio padrão (DP), coeficiente de variação (CV), assimetria (Ass) e curtose (Cur) nos diferentes momentos avaliados (Mom) de valores de umidade do solo de um Nitossolo Vermelho.

\begin{tabular}{|c|c|c|c|c|c|c|c|c|c|}
\hline \multirow{2}{*}{ Mom } & \multirow{2}{*}{ data } & \multirow{2}{*}{ Cob } & Méd & Med & Mín & Máx & \multirow{2}{*}{$\begin{array}{l}\mathrm{CV} \\
(\%)\end{array}$} & \multirow{2}{*}{ Ass } & \multirow{2}{*}{ Cur } \\
\hline & & & \multicolumn{4}{|c|}{---------------- $\mathrm{m}^{3} \mathrm{~m}^{-3}$--------------- } & & & \\
\hline$\Theta 1$ & $04 / 03 / 16$ & Viva & 0,52 & 0,52 & 0,38 & 0,62 & 9,25 & $-0,13$ & 0,20 \\
\hline$\Theta 2$ & $22 / 08 / 16$ & Viva & 0,43 & 0,43 & 0,28 & 0,62 & 13,55 & 0,03 & 1,40 \\
\hline$\Theta 3$ & $02 / 10 / 16$ & Viva & 0,37 & 0,37 & 0,20 & 0,53 & 17,01 & $-0,25$ & 0,49 \\
\hline$\theta 4$ & 09/01/16 & Viva & 0,52 & 0,52 & 0,38 & 0,62 & 9,88 & $-0,50$ & 0,26 \\
\hline$\theta 5$ & $24 / 01 / 17$ & Viva & 0,53 & 0,54 & 0,28 & 0,64 & 11,23 & $-1,07$ & 2,80 \\
\hline$\Theta 6$ & 29/03/17 & Viva & 0,47 & 0,48 & 0,28 & 0,56 & 11,46 & $-0,95$ & 1,35 \\
\hline$\Theta 7$ & $28 / 04 / 17$ & Viva & 0,53 & 0,54 & 0,31 & 0,63 & 10,53 & $-1,07$ & 2,34 \\
\hline$\Theta 8$ & $01 / 05 / 17$ & Viva & 0,47 & 0,48 & 0,29 & 0,58 & 12,10 & $-0,67$ & 0,76 \\
\hline Ө9 & $15 / 05 / 17$ & Morta & 0,56 & 0,56 & 0,30 & 0,65 & 10,18 & $-1,32$ & 3,84 \\
\hline$\Theta 10$ & $26 / 05 / 17$ & Morta & 0,53 & 0,55 & 0,31 & 0,69 & 19,68 & $-3,13$ & 13,88 \\
\hline$\Theta 11$ & $26 / 06 / 17$ & Morta & 0,54 & 0,56 & 0,33 & 0,66 & 11,99 & $-0,97$ & 1,36 \\
\hline$\Theta 12$ & $30 / 06 / 17$ & Morta & 0,51 & 0,52 & 0,30 & 0,69 & 14,75 & $-0,76$ & 0,94 \\
\hline$\Theta 13$ & 03/07/17 & Morta & 0,50 & 0,50 & 0,31 & 0,67 & 14,58 & $-0,39$ & 0,22 \\
\hline Ө14 & 10/07/17 & Morta & 0,54 & 0,55 & 0,29 & 0,64 & 11,59 & $-0,97$ & 1,79 \\
\hline$\Theta 15$ & $17 / 07 / 17$ & Morta & 0,54 & 0,55 & 0,21 & 0,65 & 13,23 & $-1,54$ & 4,95 \\
\hline
\end{tabular}

$\mathrm{Na}$ Figura 4A são apresentados os semivariogramas experimentais construídos para as séries de valores de $\mathrm{K}$, escalonados pela variância dos dados em cada série. Para cada semivariograma foi realizado o ajuste de um modelo esférico, sendo que os valores de alcance (A) são apresentados nesta Figura. O maior valor de alcance ocorreu na condição sem cobertura vegetal (K1), no valor de 7,98 m. Durante o desenvolvimento da cultura o valor de alcance foi se reduzindo, mostrando que a presença da cultura interfere no padrão espacial 
de variação da condutividade hidráulica do solo. Ao final do ciclo (K8), o alcance estava na ordem de 1,7 a 1,8m. Após a dessecação, o valor de alcance cresceu, superando 5,0 $\mathrm{m}$ para o momento K9, após o que foi se reduzindo, tendendo a estabilizar em torno de 3,0 m.
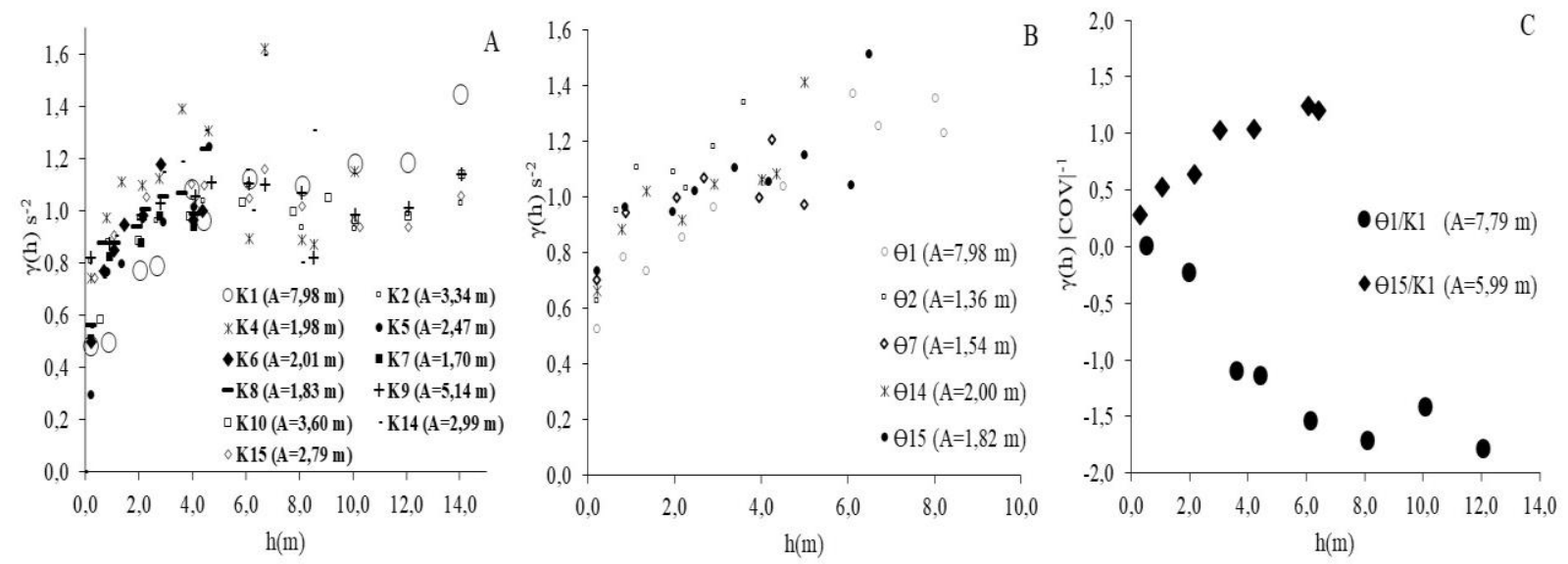

Figura 4. Semivariogramas escalonados para os i-ésimos momentos de avaliação da condutividade hidráulica saturada " $K$ " (A) e semivariogramas escalonados para umidade do solo " $\Theta$ " (B) e semivariograma cruzado de $\mathrm{K}$ com $\Theta(\mathrm{C})$ obtidas no Nitossolo Vermelho. Os termos " $\mathrm{h}$ " $\mathrm{e}$ "A" apresentados nas figuras correspondem respectivamente aos valores de distância do semivariograma e alcance do modelo esférico ajustado ao semivariograma.

$\mathrm{Na}$ Figura 4B são apresentados os semivariogramas experimentais escalonados para umidade do solo $(\Theta)$, para as séries abordadas. Continuidade espacial, expressa por um semivariograma estruturado, foi identificada apenas para as séries $\Theta 1, \Theta 2, \Theta 7$, Ө14 e $\Theta 15$. Para as demais foi identificado efeito pepita puro. Assim como obtido para condutividade hidráulica, foi encontrado $\mathrm{o}$ maior valor de alcance do modelo do semivariograma para a condição de solo anterior à cobertura vegetal, seguido de uma redução de valores à medida que houve o crescimento da cultura ( $\Theta 2$ e $\Theta 7)$. Resultados semelhantes são encontrados nos trabalhos de Vieira et al. (2010) e Hara et al. (2019).

Os semivariogramas cruzados entre $\mathrm{K} \mathrm{e}$ $\Theta$ são apresentados na Figura 4 C. Nessa figura observa-se que houve uma correlação direta entre K1 e $\Theta 1$, sendo que essa relação é esperada uma vez que a condutividade hidráulica apresenta relação funcional com a umidade do solo (VAN GENUCHTEN, 1980). $\mathrm{O}$ valor de A foi de 7,79 $\mathrm{m}$; 0,2 m menor que encontrado para os semivariogramas de cada variável, corroborando a estreita relação entre elas. Ao observar a correlação espacial entre $\mathrm{K} 1$ e $\Theta 15$, foi encontrado uma relação inversa, comprovando que houve uma inversão no padrão espacial da umidade do solo do momento $\Theta 1$ para $\Theta 15$, ou seja, de uma condição sem a presença da cultura para uma condição após o ciclo da cultura de Urochloa. Portanto, esses resultados corroboram que o sistema radicular da cultura modifica também de modo significativo o padrão espacial da umidade do solo.

Figura 5A pode-se verificar que o alcance do semivariograma decresce proporcionalmente ao desenvolvimento da cultura (MIGUEL et al., 2009), o que pode ser descrito por um modelo logarítmico, tendendo à estabilização em torno de dois metros. Isso evidencia que a estrutura de dependência espacial é drasticamente modificada ao longo do tempo no início do desenvolvimento da cultura, contudo ao decorrer do tempo ocorre a persistência de um único padrão espacial. 

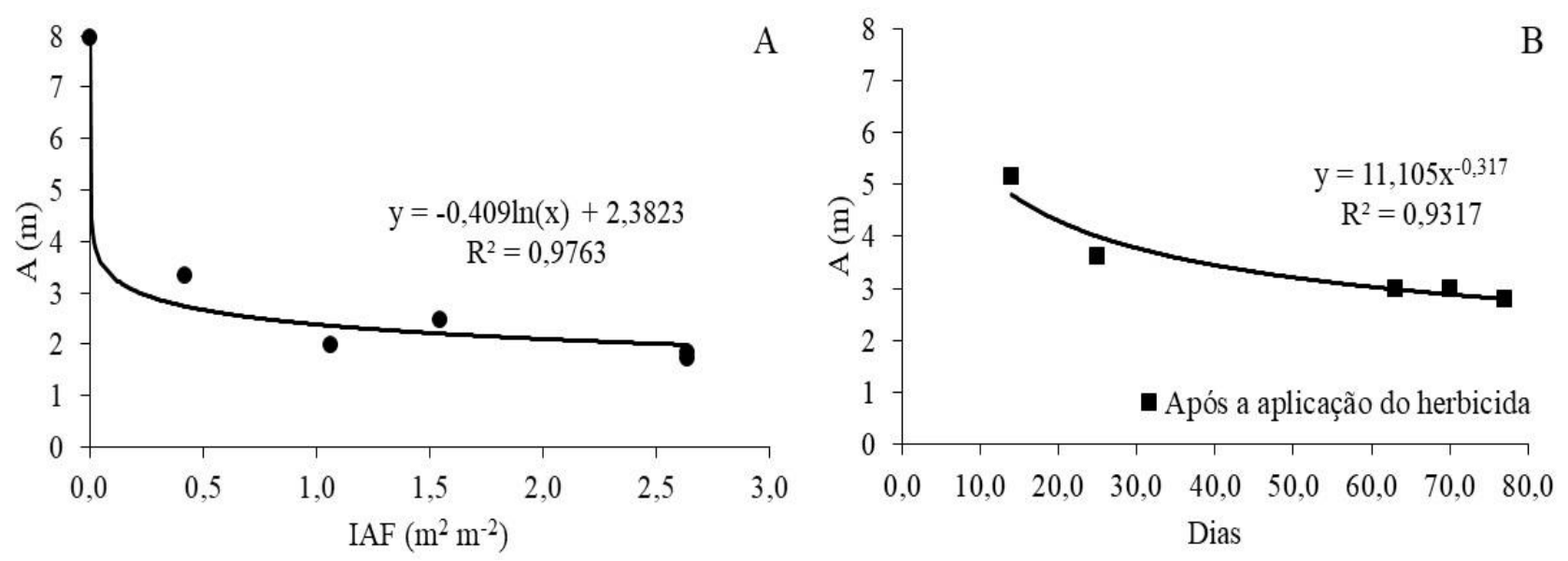

Figura 5. Alcance do modelo ajustado "A" ao semivariograma em função do índice de área foliar (IAF) (A) e alcance do modelo ajustado ao semivariograma em função do número de dias decorrentes à aplicação do herbicida (B).

Na Figura 5B pode-se verificar que no período após a dessecação, o alcance inicialmente assumiu valor mais elevado e, em seguida, também tende a se reduzir, o que pode ser descrito por um modelo potencial, tendendo a estabilização em torno de três metros. Constata-se que a cultura interage com o solo por meio do seu sistema radicular, alterando a magnitude de valores de condutividade hidráulica e alterando também o seu padrão de continuidade espacial, por meio da redução do alcance do semivariograma. Isso se deve ao fato que no desenvolvimento do sistema radicular pode promover a formação de macroporos no solo por meio de sua morte (BEVEN; GERMANN, 1982), aumento da continuidade de poros (SCHOLL et al., 2014) e aderência das partículas do solo (GHESTEM et al., 2011), culminando em mudanças nos valores da condutividade hidráulica saturada (BARTENS et al., 2008; ALLETTO et al., 2015). Na Figura 6A são apresentados os semivariogramas cruzados escalonados de $\mathrm{K}_{\mathrm{i}}$ com $\mathrm{K}_{\mathrm{i}+1}$ (A). A presença de estrutura espacial nos semivariogramas cruzados comprova a persistência temporal do padrão espacial quando as avaliações são próximas. As superfícies de valores para as séries $\mathrm{K} 6, \mathrm{~K} 7 \mathrm{e}$ K8 foram obtidas por krigagem e são apresentadas na Figura 6B. Pode-se verificar que os semivariogramas cruzados apresentam continuidade espacial que pode ser caracterizada como moderada a forte (CAMBARDELLA et al., 1994), uma vez que o efeito pepita corresponde a valores abaixo de $60 \%$ do patamar (Figura 6A).
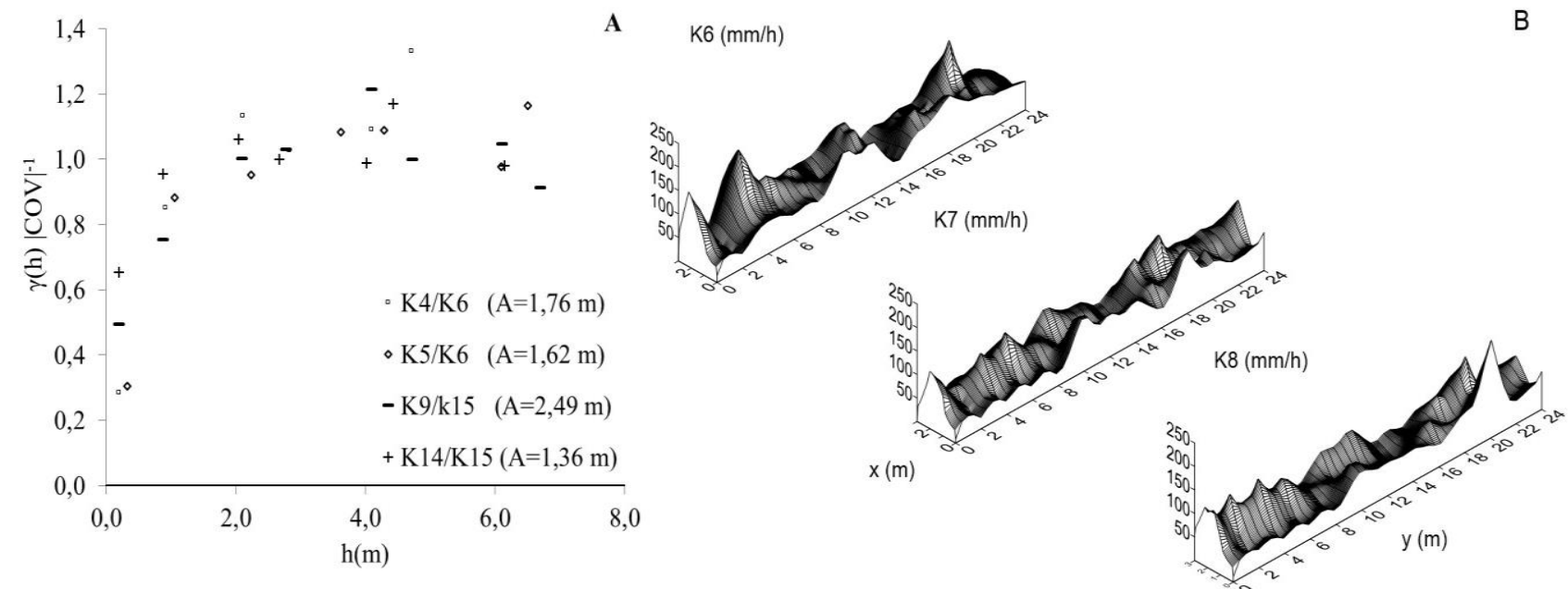

Figura 6. Semivariogramas cruzados escalonados de $K_{i} \operatorname{com} K_{i+1}(A)$ e superfície de valores de K6, K7 e K8 (B). 
As superfícies de valores mostradas na Figura 6B permitem constatar que a distribuição espacial de valores de condutividade hidráulica para a série K6 apresenta-se semelhante ao que se tem em K7 e $\mathrm{K} 8$, porém estas duas, entre si, são mais semelhantes, o que demonstra as alterações promovidas pela cultura sobre os valores e sobre o padrão de distribuição espacial da condutividade hidráulica do solo.

\section{CONCLUSÕES}

A interação do sistema radicular da cultura com a camada de solo explorada modificou as suas características, fazendo com que a condutividade hidráulica saturada se tornasse três vezes maior após o ciclo da cultura.

Durante o ciclo da cultura os valores de condutividade hidráulica crescem com o crescimento do índice de área foliar até um valor máximo, quando então começam a reduzir, em função de obstrução de parte da macroporosidade por raízes. Quando a cultura é removida, os valores retornam ao nível máximo.

Umidade e condutividade hidráulica saturada do solo apresentaram continuidade espacial de moderada a forte, e este padrão espacial foi alterado pela ação do sistema radicular da cultura, reduzindo o alcance do semivariograma ao longo do período de desenvolvimento da cultura.

As modificações promovidas pela cultura persistem no tempo, com pequenas alterações, mesmo após a remoção da cultura.

\section{REFENRÊNCIAS BIBLIOGRÁFICAS}

ALLETTO, L.; POT, V.; GIULIANO, S.; COSTES, M.; PERDRIEUX, F.; JUSTES, E. Temporal variation in soil physical properties improves the water dynamics modeling in a conventionally-tilled soil. Geoderma, v. 243244, p. 18-28, 2015. https://doi.org/10.1016/j.geoderma.2014.12.00 6

BAGARELLO, V.; IOVINO, M.; ELRICK, D. A Simplified Falling-Head Technique for
Rapid Determination of Field-Saturated Hydraulic Conductivity. Soil Science Society of America Journal, v. 68, n. 1, p. 66, 2004. https://doi.org/:10.2136/sssaj2004.6600

BARTENS, J.; DAY, S. D.; HARRIS, J. R.; DOVE, J. E.; WYNN, T. M. Can Urban Tree Roots Improve Infiltration through Compacted Subsoils for Stormwater Management?. Journal of Environment Quality, v. 37, n. 6, p. 2048, 2008 . https://doi.org/:10.2134/jeq2008.0117

BEVEN, K.; GERMANN, P. Macropores and water flow in soils. Water Resousces Reserch, v.18, n. 5, p.1311-1325, 1982. https://doi.org/10.1029/WR018i005p01311

BISWAS, A.; SI, B. C. Spatial relationship between soil hydraulic and soil physical properties in a farm field. Canadian Journal Science, v.89, n. 4, p. 473-488, 2009. https://doi.org/ 10.4141/cjss08052

BLAINSKI, E. GONÇALVES, A. C. A.; TORMENA, C. A.; FOLEGATTI, M. V.; GUIMARÃES, R. M. L. Intervalo hídrico ótimo num nitossolo vermelho distroférrico irrigado. Revista Brasileira de Ciência do Solo, v. 33, p. 273-281, 2009. https://doi.org/ 10.1590/S0100-06832009000200005

BODNER, G.; LEITNER, D.; KAUL, H.-P. Coarse and fine root plants affect pore size distributions differently. Plant and Soil, v. 380 , n. 1, p. 133-151, 2014. https://doi.org/10.1007/s11104-014-2079-8

CAllegaro, G. F. M. Compactação e infiltração de água no solo e a produtividade de milho. 2017. 50 f. Dissertação (Mestrado Profissional em Agricultura de Precisão)Universidade Federal de Santa Maria, Santa Maria.

CAMBARDELLA, C.A.; MOORMAN, T.B; NOVACK, J.M; PARKIN, T.B; KARLEN, D.L; TURCO R.F.; KNOPKA, A.E. Field-scale variability of soil proprieties in central Iowa soils. Soil Science Society America Journal, 
v.58,

p.1240-1248,

1994.

https://doi.org/10.2136/sssaj1994.0361599500 5800050033x

COSTA, N. R.; ANDREOTTI, M.; MASCARENHAS LOPES, K. S.; YOKOBATAKE, K. L.; FERREIRA, J. P.; PARIZ, C. M.; BATISTA B, C. S.; LONGHINI, E. V. Z. Atributos do solo e acúmulo de carbono na integração lavourapecuária em sistema plantio direto. Revista Brasileira de Ciência do Solo, v. 39, n. 3, p. 852-863, 2015. https://doi.org/ $10.1590 / 01000683$ rbcs20140269

GHESTEM, M.; SIDLE, R. C.; STOKES, A. The Influence of Plant Root Systems on Subsurface Flow: Implications for Slope Stability. BioScience, v. 61, n. 11, p. 869-879, 2011. 10.1525/bio.2011.61.11.6

https://doi.org/

GONÇALVES, A. D. M. A.; LIBARDI, P. L. Análise da determinação da condutividade hidráulica do solo pelo método do perfil instantâneo. Revista Brasileira de Ciência do Solo, v. 37, p. 1174-1184, 2013. https://doi.org/ 10.1590/S0100-06832013000500007

HARA, A. T.; GONÇALVES, A. C. A. Estabilidade temporal da variabilidade espacial da umidade do solo obtida no campo e no laboratório. Revista Brasileira de Agricultura Irrigada, v.12, n.6, p.3069-3077, 2018. https://doi.org/ 10.7127/rbai.v12n6001028

HARA, A. T.; GONÇALVES, A. C. A.; DOMINGUES, J. V. Estrutura espacial de armazenamento de água no solo na presença de cobertura vegetal. Revista Brasileira de Agricultura Irrigada, v.13, n.2, p.3169-3179, 2019.

10.7127/rbai.v13n2001056

MIGUEL, F. R.; VIEIRA, S. R., GREGO, C. $R$. Variabilidade espacial da infiltração de água no solo sob pastagem em função da intensidade do pastejo. Pesquisa Agropecuária Brasileira, v.44, n. 11, p. 1513-1519, 2009.
http://dx.doi.org/10.1590/S0100-

204X2009001100020

MESQUITA, M. G. B. F.; MORAES, S. O. A dependência entre a condutividade hidráulica saturada e atributos físicos do solo. Ciência Rural, v. 34, n. 3, p.963-969, 2004. https://doi.org/ 10.1590/S010384782004000300052

MORADI, A. B.; CARMINATI, A.; VETTERLEIN, D.; VONTOBEL, P.; LEHMANN, E.; WELLER, U.; HOPMANS, J. W.; VOGEL, H. J.; OSWALD, S. E. Threedimensional visualization and quantification of water content in the rhizosphere. New Phytologist, v. 192, p. 653-663, 2011. https://doi.org/ $10.1111 / \mathrm{j} .1469-$ 8137.2011.03826.x

MOREIRA, W. H.; JUNIOR, E. B.; PETEAN, L. P.; TORMENA, C. A.; ALVES, S. J.; COSTA, M. A. T.; FRANCO, H. H. S. Atributos físicos de um latossolo vermelho distroférrico em sistema de integração lavourapecuária. Revista Brasileira de Ciência do solo, v. 36, p. 389-400, 2012. https://doi.org/10.1590/S010006832012000200008.

SCHERPINSKI, C.; OPAZO, M. A. U.; BOAS, M. A. V.; SANPAIO, S. C.; JOHANN, J. A. Variabilidade espacial da condutividade hidráulica e da infiltração da água no solo. Acta scientiarum Agronomy, v.32, n. 1, p. 7-13, 2010.

https://doi.org/10.4025/actasciagron.v32i1.959

JOSÉ, J. V.; REZENDE, R.; MARQUES, P. A.; A.; GONÇALVES, A. C.; SOUZA, R. S. Variabilidade espacial de variáveis físicohídricas de dois Latossolos da região noroeste do estado do Paraná. Irriga, v. 17, n. 2, p. 208219 , 2012. https://doi.org/10.15809/irriga.2012v17n2p208

SCHOLL, P.; LEITNER, D.; KAMMERER, G.; LOISKANDL, W.; KAUL, H.-P.; BODNER, G. Root induced changes of 
effective 1D hydraulic properties in a soil column. Plant and Soil, v. 381, n. 1-2, p. 193213, 2014. https://doi.org/ 10.1007/s11104014-2121-x

SOUZA, J. M.; BONOMO, R.; PIRES, F. R.; BONOMO, D. Z. Curva de retenção de água e condutividade hidráulica do solo em lavoura de café conilon submetida à subsolagem. Coffe Science, v. 9, n. 2, p. 226-236, 2014.

TRINTINALHA, M. A. Utilização da TDR para avaliação da distribuição espacial e estabilidade temporal do armazenamento de água em um nitossolo vermelho distroférrico. 2005. $98 \mathrm{f}$. Tese de doutorado Universidade Estadual de Maringá.

VAN GENUCHTEN, M. T. A closed form for predicting the hydraulic conductivity of unsaturated soils. Soil Science Society of America Journal, v.44, n.5, 1980. https://doi.org/ 10.2136/sssaj1980.03615995004400050002x

ZAREBANADKOUKI, M.; AHMED, M. A.; CARMINATI, A. Hydraulic conductivity of the root-soil interface of lupin in sandy soil after drying and rewetting. Plant and soil, v. 398, n.1, p. 267-280, 2015. https://doi.org/ $10.1007 / \mathrm{s} 11104-015-2668-1$

ZOLIN, C. A.; PAULINO, J.; MATOS, E. DA S.; MAGALHÃES, C. A. DE S.; DE ALMEIDA, F. T.; DE SOUZA, A. P.; MINGOTI, R. Perda de solo e água sob integração lavoura-floresta e em sucessão sojamilho. Pesquisa Agropecuária Brasileira, v. 51, n. 9, p. 1223-1230, 2016. https://doi.org/ 10.1590/S0100-204X2016000900022 